\title{
Scientific Papers Poster Presentations
}

\author{
Prehospital Use of Nitroglycerin \\ According to Standard Medical Orders \\ Herman LL, Koenigsberg MD, Ward $S$ \\ University of Illinois College of Medicine, Chicago North EMS System, \\ Illinois Masonic Medical Center, Chicago, Illinois
}

Objective: To quantify the usage and effects of nitroglycerin (NTG) in prehospital care from Standing Medical Orders (SMO) for treatment of cardiac chest pain and/or pulmonary edema.

Design: Retrospective review of consecutive cases with Advanced Life Support (ALS) telemetry contact, June-August 1990.

Setting: Urban, non-profit, private, regional EMS resource hospital.

Population: Of 3,880 ALS calls, 150 patients (3.9\%) received NTG.

Interventions: NTG may be administered for cardiac chest pain and/or pulmonary edema according to SMO.

Results: Of the 150 patients $(3.9 \%)$ who received NTG, 103 $(68.7 \%)$ presented with cardiac chest pain, $35(23.3 \%)$ with pulmonary edema, and $12(8.0 \%)$ with other indications. There were 71 patients $(1.8 \%)$ with either cardiac chest pain or pulmonary edema who did not receive NTG. Commonly, NTG was not administered due to prior patient use without relief or inability to establish an intravenous (IV) line. The reason(s) for not administering NTG frequently was not documented.

Reassessment of patient condition occurred in 88 patients: $78(88.6 \%)$ had repeat of blood pressure measures; $52(59.0 \%)$ of pulses; and $41(46.6 \%)$ of respiratory rates. Chest pain was relieved in $34(38.6 \%)$, and relief of dyspnea was accomplished in $7(8.0 \%)$. The severity of the chest pain decreased in $18 \mathrm{pa}-$ tients $(52.9 \%)$; six experienced complete relief, and the level of pain did not change in $16(47.1 \%)$. Dyspnea decreased in four and was unchanged in three. Additional NTG was administered to 11 patients with persistent pain, and to three with persistent dyspnea.

Following administration of NTG, the systolic blood pressure (SBP) decreased in $57(73.1 \%)$, increased in $11(14.1 \%)$, and remained unchanged in $10(12.6 \%)$. The initial mean SBP was $183.6 \mathrm{mmHg}$ and the mean values of repeated measures of SBP was $169.0 \mathrm{mmHg}$; the mean decreased $14.1 \pm 26.2 \mathrm{mmHg}$ $( \pm \mathrm{SD} ; \mathrm{p}<.01)$. One patient became bradycardic and sustained cardiac arrest.

Conclusions: NTG is underutilized, reassessment infrequently documented, and clinically significant adverse reactions are rare. Since the incidence of hypotension, bradycardia, and cardiac arrest are rare, inability to establish an IV line should not preclude the use of NTG.

\author{
The Impact of Change in Trauma Triage Criteria in \\ Prehospital Care on Payor-Mix at an Urban Trauma Center \\ Johnson K, Koenigsberg MD, Fantus RJ, Hickey $P$, Ward $S$ \\ University of Ilinois College of Medicine, Chicago North EMS System, \\ Illinois Masonic Medical Center, Chicago, Illinois
}

Introduction: In May 1989, the Chicago EMS triage criteria were revised. Concern was expressed about further trauma center attrition due to increased financial burden.

Objective: This study analyzes the payor-mix by financial class, number of hospital days utilized, and number of patients evaluated before and after institution of new triage criteria.

Design: A retrospective, cross-sectional study of patients discharged from the trauma service.

Setting: Urban, non-profit, private, regional EMS system and level I trauma center; July-September 1988 and July-September 1989.

Participants: The study included 344 consecutive patients discharged from the trauma service during the study period who underwent triage according to EMS system trauma triage criteria. Admissions to the trauma service from other sources, i.e., walked-in, police department, and inter-hospital transfer were excluded.

Intervention: Trauma triage criteria were changed; direct utilization of a physiologic scoring system was abandoned. A system combining mechanism of injury and limited physiologic parameters was expanded.

Results: The number of patients seen in the two, three-month periods doubled from 116 to 228 ( $\mathrm{p}<.05$ ) while the average length of hospitalization decreased from 13.0 to 9.7 days $(p<.05)$. Average injury severity score (ISS) remained unchanged, 16.6 in 1988 and 16.0 in 1989. The proportion of patients with identifiable third-party coverage (commercial, HMO, Medicare, or workman's compensation) was $38.8 \%$ in 1988 and $45.2 \%$ in 1989 . The percentage of patients with public aid or no identifiable third-party payor was $61.2 \%$ in 1988 and $54.8 \%$ in 1989 ( $p>10$, Power $>80 \%)$. Mortality decreased from 31.8 to $13.6 \%(\mathrm{p}<.05)$

Conclusions: Refinement of triage criteria is an important part of the evolution of any EMS system's medical control. This study suggests that such refinement results in an increase in the number of patients seen at a trauma center, and is accompanied by a decrease in the length of hospitalization and mortality rates while the level of acuity remains unchanged. Improving the triage criteria for improving patient care resulted in an increase in patient load at the trauma centers and no change in the payor-mix of these patients; therefore increasing the financial burden. 
Determining When Pediatric Intraosseous Infusion Should Be Incorporated as a Prehospital Skill: A Decision Analysis Garrison HG, Downs SM, McNutt RA, Griggs TR

School of Medicine, University of North Carolina at Chapel Hill, Chapel Hill, North Carolina

Purpose: To determine the clinical efficacy, patient volume, and program costs that justify pediatric intraosseous infusion (IO) as a routine skill in a prehospital system.

Methods: A decision-analytic model combining probabilistic and outcome values was constructed. Critical variables for the analysis were: 1) time to intravascular access; 2) success of attaining intravascular access; 3 ) clinical efficacy of IO (i.e., the percent of lives saved by IO); 4) number of patients requiring IO annually; and 5) the cost of an IO program. Program costs included training and equipment expenses. Sensitivity analysis, which evaluates the model repeatedly using different values for the critical variables, identified those values at which IO would be cost-effective.

Results: With an estimated $80 \%$ success rate for IO access within five minutes, the cost per life saved was $\$ 201,288$. This costeffectiveness ratio required a clinical efficacy of $2 \%$, an annual program cost of $\$ 2000$, and one patient per year needing IO. The cost-effectiveness was sensitive to program costs and the number of patients needing IO. This study also suggests that epidemiologic studies would require a sample of nearly 9000 patients to prove that the clinical efficacy of IO is less than the critical value identified.

Conclusions: This analysis demonstrates that a prehospital IO program has a cost of $\$ 201,288$ per life saved at a clinical efficacy of $2 \%$. While prehospital IO studies have failed to show any clinical efficacy, it is doubtful that past or future studies will have adequate statistical power to show IO has an efficacy of less than $2 \%$. Therefore, IO is likely cost-effective, but must be considered in the context of other prehospital programs. Analyses such as this permit rational prioritization of programs in prehospital systems.

\author{
Accuracy of the Prehospital Index in Identifying \\ Major Hemorrhage in Trauma Victims \\ Newman C, Jones JS, Mattice C \\ Butterworth Hospital Michigan State University College of Human \\ Medicine, Grand Rapids, Michigan
}

Purpose: The immediate recognition of life-threatening hemorrhage after injury in the prehospital and emergency department setting clearly is a critical component in the provision of optimal care for seriously injured trauma victims. To determine the sensitivity of the Prehospital Index (PHI) in identifying patients with severe blood loss, a one year review was conducted at a regional trauma facility.

Methods: The study population consisted of 217 consecutive trauma admissions (ages 3 to 88 years). Patients were managed using standard resuscitation techniques; blood transfusions were ordered at the discretion of attending physicians and did not follow any preplanned protocol. Medical records were examined to determine total blood requirements for each patient during the first 12 hours of hospitalization, the emergency department (ED) disposition, and outcome. The following clinical variables were analyzed (Student's $t$ test) to determine their value as predictors of blood loss: age; sex; mechanism of injury; initial vital signs; revised trauma score; prehospital index (PHI); and injury severity score.

Results: Forty-two percent (92 patients) received transfusions during the first 12 hours of hospitalization. The best predictor of blood loss was the prehospital index. Of the total group, $55 \%$ $(119 / 217)$ had a PHI $\leq 3 ; 86 \%(102 / 119)$ of these patients did not require transfusion. Forty-five percent of the total group had a PHI greater than $3 ; 77 \%(75 / 98)$ of these patients did require transfusion and received an average of 7.1 units of packed cells.

Conclusions: These data strongly suggest that patients with $\mathrm{PHI}$ scores $>3$ require close hemodynamic monitoring to rule out significant blood loss and may warrant immediate cross-match on arrival to the emergency department. 


\section{You Can Make A Difference}

\section{Cervical Spine Issues in Prehospital Medicine}

An educational videotape by Ron Stewart, $M D$

Every year, 13,000 Americans suffer a spinal cord injury. Of those victims, 8,000 survive thanks to advances in the study of cervical spine issues.

Dr. Stewart examines the history of spinal cord injury (SCI) and presents some startling numbers on the changing face of SCI treatment in the prehospital setting. Learn the facts about SCI and how you can make a difference.

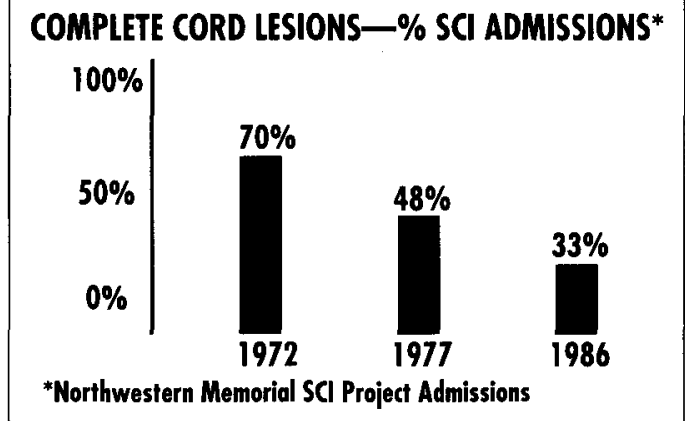

Spinal cord injury (SCI) registries reveal a consistent decline in the number of patients with complete cord lesions presenting to hospitals caring for SCI. In 1972, some 70 percent of patients presenting to major SCl centers had complete cord lesions, compared to 48 percent in 1977 and 33 percent in 1986. Many would attribute much of this decline to improved training, procedures, and equipment of prehospital care personnel.

Cervical Spine Issues in Prehospital Medicine is a non-profit videotape produced by California Medical Products in conjunction with Emergency Medical Update. Proceeds from the sale of every tape go to support research conducted by the National Association of EMS Physicians.

\section{Cervical Spine Issues in Prehospital Medicine: $\$ 49.95$}

MAll TO: The Jems Bookstore • P.O. Box 2789 • Carlsbad, CA 92018

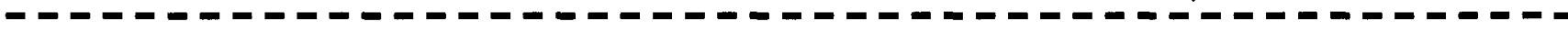

YeS. I'd like to order Cervical Spine Issues in Prehospital Medicine for \$49.95.

Name

Institution

Address

City/State/Zip

Charge My: $\square$ VISA $\square$ MasterCard

ACCOUNT NUMBER

EXPIRATION DATE

SIGNATURE

(Please add $\$ 3.00$ shipping and handling.)
Mail to: The Jems Bookstore P.O. Box 2789

Carlsbad, CA 92018

You may order by calling the Jems Bookstore, toll-free, at

1-800-266-JEMS

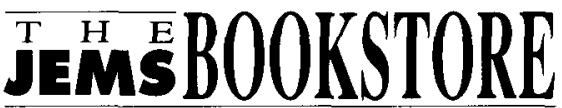


The Prehospital Use of Nebulized Albuterol for Patients with Wheezing Whose Chief Complaint is Shortness of Breath Dickinson ET, O'Connor RE, Megargel $R$

The Medical Center of Delaware, Wilmington, Delaware

The use of nebulized beta-agonists in the prehospital EMS setting has become widespread, and their safety and efficacy have been documented. Our purpose was to study their broadened use and determine their effectiveness in specific sub-sets of wheezing patients.

This 6.5 month, prospective study was conducted to determine the benefit of nebulized albuterol treatments on a variety of wheezing patients whose chief complaint to paramedics was shortness of breath.

Methods: Four groups were identified by patient history; Asthma, COPD, Asthma and COPD (A/C), and Non-Asthma/ Non-COPD (NANC). The effectiveness of the treatment was evaluated objectively by peak expiratory flow rates (PEFR) obtained before and immediately after treatment, subjectively by comparison of the paramedics' respiratory assessment before and after treatment, and by the patient's evaluation of their dyspnea. Changes in PEFR were subjected to analysis by a paired $t$ test.

Results: During the study period, 104 patients received albuterol treatments. Patients with incomplete data and those who received other prehospital medications were excluded. Sixty-two patients met all criteria.

$\begin{array}{lrrrr}\text { Group } & \mathbf{n} & \text { Age } & \begin{array}{c}\text { Change } \\ \text { PEFR (L/min) }\end{array} & \text { Subjective } \\ \text { Asthma } & 36 & 43 \pm 21 & 54(\mathrm{p}>.005) & 97 \% \\ \text { COPD } & 12 & 67 \pm 13 & 28(\mathrm{p}>.005) & 92 \% \\ \text { A/C } & 7 & 68 \pm 11 & 15 \text { (insignificant) } & 100 \% \\ \text { NANC } & 7 & 52 \pm 25 & 34(\mathrm{p}>.05) & 86 \%\end{array}$

Conclusion: Albuterol was effective in increasing the PEFR in patients with Asthma, COPD and NANC. Patients with both Asthma and COPD do not demonstrate increased PEFR after treatment. Most patients were improved subjectively after nebulized albuterol treatments.
The Prevalence of Occupational

Injuries to EMTs in New England Schwartz RJ, Benson L, Jacobs L

Hartford Hospital, Hartford Connecticut

Objective: To characterize the prevalence and morbidity of injuries to Emergency Medical Technicians (EMT) in New England

Design: Survey of a $2 \%$ random sample of all EMTs in New England. Identity remained anonymous. A second, follow-up mailing was conducted to improve return rate.

Intervention: Epidemiologic calculations of responses to questionnaire.

Results: Questionnaires were sent to 786 EMTs in Maine, New Hampshire, Vermont, Massachusetts, Rhode Island, and Connecticut, and 439 (56\%) questionnaires were returned representing 13,875 hours of duty time in six months. Male EMTs comprised $71 \%$ of the respondents. The mean age of the respondents was 35 years, and $68 \%$ were basic EMTs. Injury attack rates were: stress $11.2 \%$; back $10.5 \%$; extremity $9.8 \%$; assault $8.4 \%$; ambulance collision $4.1 \%$; hearing loss $2.5 \%$; eye $1.4 \%$; and $12 \%$ were injured more than once. Paramedics were more likely to be assaulted, be involved in a motor vehicle collision, or suffer from stress, and were less likely to have a back injury than were basic EMTs. There were minor interstate differences. Only $1 \%$ of the work force was disabled due to back injuries; four EMTs encountered loss of duty time secondary to an assault, and $0.5 \%$ of EMTs were out of work due to stress.

Conclusions: The results of this survey begin to characterize the occupational risks of EMTs. The prevalence of back injuries, assault, stress, and extremity injuries seem too high. Educational programs and preventive interventions should be designed to minimize back injuries, stress, and assault. 


\author{
A Model Instructional Strategy-for Teaching Prehospital \\ Management of Patients With Alzheimer's Disease \\ Cason D, Wilson RW, Chafetz PK, McIntire DD \\ University of Texas Southwestern Medical Center at Dallas, Dallas, \\ Texas
}

An increased incidence of Alzheimer's Disease (AD) is accompanying the graying of America and has points out the need to teach prehospital personnel how to manage these patients. The purpose of this project is to develop instructional strategy to teach prehospital personnel about $\mathrm{AD}$ and basic management techniques. This information includes: causes of acute- and gradual-onset confusion in the elderly; symptoms and course of $\mathrm{AD}$; mental status exam; methods to facilitate communication; techniques to avoid confrontation; and means to identify personal feelings about the elderly and $\mathrm{AD}$.

Teaching methodology includes instructional objectives, videotape presentation of didactic/clinical material, videotape situations demonstrating patient management techniques, roleplaying exercises, and discussion groups.

The videotape presentation portion of the teaching methodology was shown to be effective as a single instructional strategy. A total 134 EMS personnel were given a 16 -item exam before and after the videotape presentation. Pre- to post-test score difference was significant at $p=.001$. Additional instruction components may impact further on the knowledge of $\mathrm{AD}$ and positive delivery of care to the elderly.

\section{Value of Physician-Staffing at a Large Air Show Boyle MF, DeLorenzo RA Department of Emergency Medicine, Wright State University, Dayton, Ohio}

Purpose: To determine if on-site physician care is required at a large, international air show.

Methods: The Dayton Air Show is one of the largest events of its kind in North America. The summer event has an average annual attendance of 225,000 for the two-day period. Medical coverage includes 10 fully-staffed, paramedic ambulances and five aid stations staffed by nurses and emergency medicine residents with on-site attending physician supervision. Physicians were available to evaluate patients before disposition.

A retrospective chart review was performed on all medical encounters during coverage at the Dayton Air Show for the years 1988-1990. Current published guidelines recommend that certain complaints, (e.g., headaches and heat injuries), be evaluated by physicians.

Results: The records of 819 patients were abstracted. Of these, $232(28.3 \%)$ presented with minor scrapes and blisters, 179 $(21.8 \%)$ with headaches, and $97(11.9 \%)$ with heat and environmental injuries. A total of 29 patients $(3.5 \%)$ required immediate transportation to the emergency department. Physicians evaluated 790 (96.4) patients who were treated and released at the scene. One patient in the study suffered cardiac arrest.

Conclusion: Most patients were treated and released by the onsite physicians. Conservation of prehospital and transportation resources is an important physician role in mass gatherings of this type.

\author{
The Effect of Intravenous Glucose \\ Boluses on Serum Potassium Levels \\ Trueba D, Wolford $R W$ \\ University of Illinois College of Medicine at Peoria, and Central \\ Illinois Center for Emergency Medicine, Saint Francis Medical Center, \\ Peoria, Illinois
}

Purpose: To determine if intravenous (IV) glucose boluses cause significant alterations of serum potassium $\left(\mathrm{K}^{+}\right)$levels.

Methods: A prospective, descriptive study of patients $\geq 18$ years of age presenting with altered levels of consciousness (ALOC) to paramedics in the prehospital setting or to a community teaching hospital ED who received 50\% Dextrose (D50) intravenously (IV). At presentation, a blood sample (PRE) was obtained prior to D50 therapy. For patients treated by paramedics, a second blood sample (POST) was obtained upon emergency department (ED) arrival. For patients seen initially in the ED, the POST sample was obtained one hour after D50 therapy. Both samples were analyzed to determine $\mathrm{K}^{+}$and glucose levels. Results: Over a five-month period, 35 patients met study criteria. The average age was $46 \pm 20$ years. The study found that 22 of 35 patients $(63 \%)$ had PRE blood sugars (BS) $<80 \mathrm{mg} / \mathrm{dl}$ (mean PRE BS was $37 \pm 13 \mathrm{mg} / \mathrm{dl}$, mean POST BS was $140 \pm 47$ $\mathrm{mg} / \mathrm{dl}$ ) and $13 / 35(37 \%)$ had a PRE BS $>80 \mathrm{mg} / \mathrm{dl}$, (mean PRE BS was $261 \pm 280 \mathrm{mg} / \mathrm{dl}$, mean POST BS was $375 \pm 264$ $\mathrm{mg} / \mathrm{dl}$ ). The average PRE $\left[\mathrm{K}^{+}\right]$was $4.1 \pm 0.8 \mathrm{mEq} / \mathrm{L}$ and the average POST $\left[\mathrm{K}^{+}\right]$was $4.1 \pm 0.7 \mathrm{mEq} / \mathrm{L}$. One-third $(12 / 35)$ of the patients had a $<5 \%$ change in $\left[\mathrm{K}^{+}\right], 8 / 35$ patients $(23 \%)$ had a $>5 \%$ increase in $\left[\mathrm{K}^{+}\right]$, and $10 / 35$ patients $(29 \%)$ had a $>5 \%$ decrease in $\left[\mathrm{K}^{+}\right]$. The administration of $\mathrm{D} 50$ resulted in a $>20 \%$ increase in $\left[\mathrm{K}^{+}\right]$in $4 / 35$ patients $(11 \%)$ and only $1 / 35$ $(3 \%)$ had a $>20 \%$ decrease in $\left[\mathrm{K}^{+}\right]$.

Conclusion: Boluses of intravenous glucose produce unpredictable changes in serum $\left[\mathrm{K}^{+}\right]$. For most patients, these changes probably are insignificant clinically. However, these are preliminary data from an ongoing clinical study. 
Rectal Diazepam for Prehospital Pediatric Status Epilepticus Dieckman R, Pointer JE, Barton C, Sullivan P, Hayden C San Francisco General Hospital; Medical Director, San Francisco City and County EMS; Moffitt Hospital, University of Califormia, San Francisco; Children's Hospital of San Francisco; Kaiser Permanente Medical Center, San Francisco, California

Introduction: Seizures are a common pediatric prehospital emergency. Most are brief and do not require treatment. A small proportion progress to status epilepticus (SE); this group often presents vexing field problems, especially in establishment of vascular access and from respiratory depression secondary to parenteral anti-convulsant administration. Recent studies have shown poor correlation of duration of SE with neurologic outcome. Therefore, a slow-acting, first-line, anticonvulsant, with a high therapeutic ratio is needed. One such agent is rectally administered diazepam (RD), which has been used safely in the field environment.

Methods: This study analyzed paramedic use of RD in the prehospital treatment of pediatric SE over a 12-month interval. Protocol allowed administration of $0.3-0.5 \mathrm{mg} / \mathrm{kg}$ of parenteral diazepam solution, per high rectal tube, after 15 minutes of observed seizures.

Results: There were 140 cases of seizures in children under 18 years of age during the study period, representing $34 \%$ of nontrauma-related pediatric cases called to the base-hospital. There were 17 patients that met $\mathrm{SE}$ criteria (median age $=3$ years). Ten patients received $\mathrm{RD}$; six received intravenous (IV) diazepam; and one received both. Of the 11 patients treated with $R D$, nine stopped seizing prior to hospital arrival. Both patients who failed to stop seizing following administration of $\mathrm{RD}$ required extensive emergency department (ED) management, including multiple anti-convulsants and endotracheal intubation. Both had encephalitis. No patient in the RD group who stopped seizing experienced a significant complication.

Conclusion: This study suggests that RD is a safe, easy-to-use, efficacious treatment perfectly suited for prehospital pediatric SE.
The Impact of a Physician-Staffed Advanced Life Support Unit on Prehospital Intervention Robey W, DiLeonardo F, Cody $R$, Sierra $N$ Morristoun Memorial Hospital. Morristown New Jersey; Robert Wood Johnson Medical School, Piscataway, New Jersey

Objective: To determine the impact of an on-scene physician upon prehospital interventions.

Methods: A retrospective chart review was conducted of 610 advanced life support (ALS) dispatches in a suburban area of northwestern New Jersey. This two-tiered system consists of basic life support (BLS) ambulances and independent ALS vehicles. There were $374(61 \%)$ ALS runs staffed only by paramedics, and 236 (39\%) were staffed additionally by a senior, PGY-III Emergency Medicine resident. Data collected included: the ALS versus BLS triage decision as determined by the basestation or on-scene physician; on-scene times; and the number and types of procedures and medications administered. Data were evaluated using chi-square and Wilcoxon Rank Sum tests. Results: At least one ALS intervention was ordered in $\mathbf{4 6 5}$ $(76 \%)$ of dispatches. The only statistically significant difference in the parameters studied was in on-scene times, i.e., $14.9 \mathrm{~min}$ utes and 16.8 minutes, with and without a physician present, respectively $(p=.01)$. No physician-specific procedures (cricothyrotomy, central venous access, venous cutdown, tube thoracostomy) were performed despite the availability of this capability. Conclusion: The presence of an on-scene physician did decrease on-scene times by a statistically significant 1.9 minutes. However, based on these results, a physician-staffed ALS unit may not have a clinically significant effect on prehospital care. 


\author{
Adequacy of Ventilation Using an \\ AIDS-Virus-Impermeable Airway \\ Lufkin K, Ruiz E \\ Hennepin County Medical Center, Minneapolis, Minnesota
}

Hypothesis: An AIDS virus impermeable airway (Kiss of Life, [KOL]), designed to prevent contamination of the rescuer, permits adequate mouth-to-airway ventilation in cardiopulmonary arrest.

Methods: Ten adult patients who failed to respond to standard CPR in the emergency department (ED) were studied. Those with severe lung disease or suspected pulmonary embolism were excluded. Upon declaration of death (time $=0$ ), blood gases were drawn from radial artery lines while closed cardiac massage continued, and endotracheal and gastric tubes were removed. Subsequently, the KOL airway was placed and room air, mouth-to-mouth ventilation was begun at the rate of 12 breaths/minute. Arterial $\mathrm{pH}, \mathrm{pCO}_{2}$, and $\mathrm{pO}_{2}$ were measured every two minutes for a maximum of 10 minutes.

Results: Good breath sounds were achieved easily in seven of the ten patients. The $\mathrm{PaCO}_{2}$ range after 10 minutes in six of these seven was 33 to 49 torr. The seventh had a $\mathrm{PaCO}_{2}$ of 108 torr at time 0 . The remaining three patients were not well-ventilated. Two of these were edentulous and the last had required transtracheal needle ventilation during attempted resuscitation.

Conclusion: This pilot study shows that patients in cardiopulmonary arrest may be ventilated adequately with the KOL airway if they are not edentulous. This airway, with previously demonstrated features that minimize contamination, is attractive particularly to AIDS-conscious, first responders.

\author{
National Trends in Staffing Advanced \\ Life Support Systems in the Private Sector \\ Hunt DM, Socher $M M$ \\ The George Washington University Medical Center, Washington, D.C.
}

Purpose: Very little literature is available that describes staffing patterns in emergency medical services (EMS). To discern national trends in the private-sector staffing patterns, a nationwide survey was conducted of private companies that provide emergency medical services in the 200 most populous cities in the United States.

Methods: A two-page questionnaire was mailed to 117 services comprising three major categories-private, hospital-based, and public utility model (PUM) services. There were 36 organizations that responded to the first mailing, while a second mailing generated an additional 17 responses. This provided a $45 \%$ overall response.

Results: Responses from $56 \%$ of the services that returned the survey supported the use of two paramedics, as opposed to one paramedic and one emergency medical technician (EMT). Flexible, peak-load staffing is used by $67 \%$, though $33 \%$ continue to use classic, fixed shifts. System Status Management (SSM) is employed by 30 of the 53 respondents.

Conclusions: Due to the severe nationwide shortage of paramedics, EMS systems have resorted increasingly to creative staffing solutions. Flexible scheduling increases the use of parttime personnel. Together with peak-load staffing, this offers the private services a larger, more diverse labor pool enabling them to compete for employees with the more traditional fire services. As companies attempt to contain costs, the increased use of more sophisticated, computerized dispatch systems and SSM has grown in popularity. Privatization is maintaining its share of the EMS market. 
Diminishing Stretcher-Related Back Injuries in EMS Personnel Meskill DJ, Curka PA, Pepe EP, Steich WG, Gray D Baylor College of Medicine, The Cily of Housion Fire Department, EMS Division, Houston, Texas

Purpose: Previous studies have demonstrated the high incidence and expensive consequences of occupationally-related back injuries (OBI) in EMS personnel, primarily associated with the loading and unloading of patient-stretchers. The purpose of this study was to see if the introduction of a speciallydesigned stretcher could diminish the relative occurrence of this type of OBI.

Methods: All personal injury incidents reported for the 12 months before the introduction of the new stretchers (FernoWashington Model 35-A) were compared to a 12-month period following their installation. The study was conducted in a single, municipal EMS system in a busy, urban center (over 140,000 EMS incidents/year). All personnel received directly supervised training in the proper use of the new equipment.

Results: Although the total number of EMS incidents concurrently had risen from 120,000 to over 140,000 /year, OBI in EMS personnel specifically related to the loading and unloading of patients into and out of ambulances fell by $60 \%$, from 47 to 19 per year. Overall, following introduction of the new device, the incidence of stretcher-related OBI relative to the total number of reported EMS-related, back injuries diminished significantly ( $\mathrm{p}=0.002$ by chi-square) from $86 / 126(68 \%)$ to $69 / 185(37 \%)$.

Conclusion: Specially-designed stretchers can diminish the incidence (and accompanying high cost) of certain stretcher-related back injuries known to occur in EMS personnel. However, one other factor, the accompanying in-service training also may play a key contributing role.
Hypokalemia Associated with Albuterol Delivered by Nebulizer or Metered Dose Inhaler with Spacer Idris AH, McDermott MF, Morrabel A, RaucciJ

University of Florida College of Medicine, Gainesville, Florida; Cook County Hospital, Chicago, Illinois; University of Illinois College of Medicine, Chicago, Illinois

Hypothesis: Plasma potassium concentrations decrease significantly in patients treated for acute asthma with albuterol regardless of whether the drug is given by nebulizer or metered dose inhaler with spacer (MDIS).

Methods: A total of 21 patients with acute asthma were evaluated in a prospective, randomized, double-blinded, multi-center study. Data analysis was accomplished using the two sample $t$-test, with statistical significance set at $\mathrm{p}<.05$. Patients were treated every 30 minutes with four puffs of placebo MDIS, followed by nebulized albuterol $2.5 \mathrm{mg}$ in $2 \mathrm{ml}$ of normal saline or with 4 puffs of MDIS albuterol followed by placebo inhaler ( 2.5 $\mathrm{ml}$ normal saline). Samples of blood for potassium and theophylline levels were drawn before the first treatment and for potassium concentration after the last treatment.

Results: The mean $( \pm 1$ sd) plasma potassium concentration was $4.0 \pm 0.41 \mathrm{mEq} / \mathrm{L}$ before treatment. After treatment plasma potassium decreased by a mean of $0.40 \pm 0.26 \mathrm{mEq} / \mathrm{L}(\mathrm{p}<.0001)$. The mean decrease in plasma potassium for the nebulizer and MDIS groups, respectively, was $0.47 \pm 0.31 \mathrm{mEq} / \mathrm{L}$ and $0.33 \pm 0.16$ $\mathrm{mEq} / \mathrm{L}$ (NS, $\mathrm{p}=.20$; power=.82, 0.3 difference of means). In patients who had one to three doses or four to six doses of albuterol, respectively, the mean plasma potassium decreased by $0.41 \pm 0.32$ and $0.40 \pm 0.18 \mathrm{mEq} / \mathrm{L}(\mathrm{NS}, \mathrm{p}=.94)$.

Conclusion: Both nebulized and MDSI albuterol caused a significant decrease in plasma potassium, but the decrease was not significantly different for either delivery method, nor for low or high albuterol dosage. 


\author{
Paramedic Diagnostic Accuracy in Patients \\ with Chest Pain or Shortness of Breath \\ Schaider J, Riccio J, Rydman R, Pons $P$ \\ Cook County Hospital, University of Mllinois College of Medicine; \\ Denver General Hospital, University of Colorado, Boulder, Colorado
}

Objective/Hypothesis: Thrombolytic therapy in the prehospital setting reduces the time to coronary reperfusion. The ability of paramedics to identify patients who qualify for thrombolytic therapy needs further evaluation. This study was undertaken to determine the accuracy of the paramedic diagnosis in patients transported with a chief complaint of chest pain or shortness of breath.

Design: In this prospective, cross-sectional study, paramedics evaluated the patient and then completed a standard form indicating the patient organ system diagnosis and specific diagnosis. The paramedic and emergency department diagnoses were compared to assess the accuracy of the paramedic diagnosis.

Setting: Multi-hospital: one large, municipal hospital and one community hospital.

Participants: The study included all patients that complained of chest pain or shortness of breath who were transported to the study centers via the City of Denver paramedics during a twomonth period. Of the 102 patients entered, 98 completed the study.

Interventions: None.

Results: Diagnostic concordance data were analyzed by organ system and specific diagnoses within organ systems using the kappa statistic. Statistically significant agreement was found for cardiac and pulmonary organ systems. For specific diagnoses within organ systems, all observed levels of agreement were less than were those of organ system diagnoses $(p<.05)$. For specific diagnoses, significant agreement was found for cardiac cases $(\mathrm{p}<.00006)$, and non-concordance was identified for idiopathic cases $(\mathrm{p}<.0008)$.

Conclusions: Overall, paramedics have excellent diagnostic accuracy when compared to emergency department diagnosis by organ system $(82 \%)$. They obtained very good agreement for specific cardiac diagnoses $(77 \%)$.
Discrepancy between Palpated and

Auscultated Systolic Blood Pressures

Doctor A, Wolfson A, Menegazzi J, Platt T

University of Pittsburgh Affiliated Residency in

Emergency Medicine, Pittsburgh, Pennsylvania

Purpose: To determine whether there is any difference between palpated and auscultated systolic blood pressures (BP) in young, healthy individuals when measured by emergency medical technicians (EMTs).

Methods: In this randomized, blinded study, 40 EMTs (27 male, 13 female; mean age $=26$ years; mean EMS experience $=3.5$ years) participated. Subjects were classified into groups of five. At random, each subject took one sitting systolic BP measurement in the right arm, either palpated or auscultated, on each of the other subjects in the group. Thus, each subject also had his or her own BP measured once by each of the other subjects in the group (twice by palpation and twice by auscultation). Measurements on two subjects were excluded because of protocol violations. A total of $152 \mathrm{BP}$ measurements were recorded. Mean \pm 1 sd values were calculated for palpated and auscultated BP and compared by two-tailed $t$-test for paired samples. Individual measurements were compared by repeated measures ANOVA, and Tukey's test, with alpha error set at .05.

Results: Mean BP by palpation was $114 \pm 11 \mathrm{mmHg}$, compared to $120 \pm 11 \mathrm{mmHg}$ by auscultation $(\mathrm{p}=.0000006)$ with a mean difference of $6.3 \mathrm{mmHg}$ (95\% CI: 4.2 to 8.4 ). In only six of the 38 subjects was the mean palpated BP higher than auscultated BP ( $<<.00003$ by one-sample exact binomial test, assuming $50 \%$ chance distribution).

Conclusions: When systolic BP measurements are performed by EMTs on young healthy individuals, palpated BPs are lower than auscultated BP by a mean of $6.3 \mathrm{mmHg}$. Although this discrepancy may not affect clinical decision-making in normotensive patients, further research is required to determine whether the accuracy of palpated BPs would decrease in hemodynamically unstable patients. This possibly could affect prehospital decision-making. 


\section{Methodology for Comparative Testing of Prehospital Devices \\ DiLorenzo RA, Popky $L M$ \\ University of California Irvine Medical Center, Orange, California}

Purpose: Performance standards for prehospital emergency medical care devices are either non-existent or derived from inhospital settings. This paper proposes a methodology to compare such devices in a way that recognizes the unique features of the prehospital care environment.

Methods: As a model, a study of portable suction devices was chosen. In the initial phase, prehospital providers nation-wide were surveyed regarding their experience with these devices. The survey addressed scope of need, availability, performance, durability, training, maintenance, safety, and the relative importance of each of these issues. The results of the survey were used to design a field simulation as well as to develop a weighted scoring system. In the second phase, eight of the most widely used devices were studied by standard, in-hospital criteria (e.g., peak pressure, airflow rate), as well as in simulated field use (e.g., suction of simulated mucous and emesis).

Results: Field simulations clearly yielded different preferred devices than did in-hospital criteria. The weighted scoring system further differentiated the suction devices.

Conclusion: This study indicates that in-hospital performance standards are insufficient to use to judge prehospital care equipment. A methodology is proposed for comparative testing devices using a provider survey and field use simulations to reflect more accurately the unique nature of the prehospital environment. Based on this methodology, local EMS system variations will dictate the choice of different devices.

\author{
Inaccuracy of Oxygen Flow Meters in an Urban EMS System \\ Michelson E, Echegaray RF, Schneider $S$ \\ University of Pittsburgh, School of Medicine, Montefiore University \\ Hospital, Pittsburgh, Pennsylvania
}

Hypothesis: Oxygen therapy is dependent on rate of gas delivery by oxygen flow meters (OFM). Some patients with emphysema are very sensitive to the administration of supplemental $\mathrm{O}_{2}$. Hand-held nebulizer function also varies with $\mathrm{O}_{2}$ flow rate. The OFMs are assumed to be accurate and not routinely tested. This study measured the accuracy of a sample of OFMs in use in an urban EMS setting.

Methods: There were 28 OFMs randomly chosen for measurement, including eight different models in use in five EMS ambulances and three urban teaching hospital emergency departments (ED). Flow rates were determined at 2, 4, 6, and 8 $\mathrm{L} / \mathrm{min}$ settings by timing displacement of $6 \mathrm{~L}$ of water from an inverted 6L Erlenmeyer flask. Multiple measurements were averaged for each flow meter at each rate setting. Wall $/$ tank $\mathrm{O}_{2}$ pressure was recorded for each OFM. Ambulance OFMs were tested using the vehicle oxygen system.

Results: A different OFM was the most or least accurate at each setting. There were five samples of one OFM model which had a mean and median error of $2.5 \%$ and $1 \%$ at $2 \mathrm{~L} / \mathrm{min}$. Only 4 of $18(22 \%)$ OFMs in use in the ED had $<10 \%$ error at all three settings. The five EMS ambulances tested all had oxygen systems that exceeded 50 psi at the wall outlet, yet the EMS OFMs were more accurate at $2 \mathrm{~L} / \mathrm{min}$ than were the ED OFMS. Errors were comparable at higher flow rates. Only five OFMs under-delivered $\mathrm{O}_{2}$, four of those five only at $2 \mathrm{~L} / \mathrm{min}$.

Conclusion: A sample of OFMs in an urban EMS system produced variably inaccurate oxygen flows. Most OFMs delivered excess oxygen. Portable $\mathrm{O}_{2}$ tank regulators were the least accurate. Routine testing of OFMs for accurate gas delivery may be indicated. The clinical significance of oxygen delivery in accuracy remains to be determined. 
Tactical Emergency Medical Support (TEMS): An Emerging Specialized Area of Prehospital Care Carmona R, Rasumoff D

Department of Trauma Services, Tucson, Arizona; Department of Emergency Medicine, County USC Medical Center, Los Angeles, California

Purpose: Prehospital Tactical Emergency Medical Support (TEMS) of Police High Risk Operations such as Special Weapons and Tactics (SWAT) Teams is increasing nationally. No information currently is available as to the scope of TEMS or relative to the epidemiology of the problems encountered. This study surveys the practice of TEMS nationally and provides recommendations for training and standardization of TEMS.

Methods: Nationally, 81 SWAT teams were surveyed as to their activities over a 3 year period. Information included, but was not limited to, the number of tactical operations, type of TEMS used, emergency medical conditions encountered, secure or not-secure tactical environment when TEMS was required, and general information such as the types of training received and agencies the TEMS providers represented.

Results: All teams had, at a minimum, EMT-level providers at a safe, tactical outer perimeter location. Paramedics were utilized in $58 \%$ of the programs, with $42 \%$ of those able to function in an environment that is not secured tactically. Of the prehospital providers, $35 \%$ were full-time or reserve police officers. All providers had some tactical training with $47 \%$ having attended a formal SWAT school. There were 79 injuries and 35 deaths reported. Gun shots were not uncommon and explosive devices commonly were used. Medical emergencies occurred infrequently. Medical control was via telemetry with $87 \%$ of the teams, while $12 \%$ had on-scene physician control. Two teams have physicians who are police officers, one of which is SWAT trained and qualified.

Conclusions: TEMS is required for high-risk police operations for improved prehospital care and eduction in risk and liability.

\author{
Estimated Cost-Effectiveness of Dispatcher \\ CPR Instruction via Telephone to Bystanders \\ in Out-Of-Hospital Ventricular Fibrillation \\ Valenzuela TD, Clark LL, Spaite DW, Criss EA, Meislin H, Sayre RO \\ Arizona Medicine Research Center, University of Arizona College of \\ Medicine and Tucson Fire Department
}

Hypothesis: Emergency CPR instruction via telephone (ETCPR) is cost-effective compared to prehospital EMT/ paramedic treatment alone for witnessed ventricular fibrillation (VF) in adult patients.

Methods: A total of 118 patients, age $>18$ years, with prehospital, witnessed VF were studied over 22 months. Patient data were extracted from hospital records, monitor-defibrillator recordings, paramedic reports, dispatching records, and telephone interviews with bystanders. No ETCPR was available during this period. The costs of ETCPR implementation were estimated retrospectively. The marginal cost of the paramedic service attributable to treatment of VF was calculated from fire department records. Years-of-life-saved were estimated from age, gender, and race-matched norms. Means were tested for significance by student's $t$-test.

Results: Of the patients receiving bystander CPR (BCPR), 14 of 32 (26\%) patients survived to hospital discharge versus 4 of 65 patients $(6 \%)$ who did not have BCPR. These groups did not differ significantly $(p<.05)$ in age, EMS response times, or time from collapse to defibrillation. Time from collapse to CPR was significantly less in patients with BCPR (1.8 min) versus patients without $B C P R(7.1 \mathrm{~min})$. Had all patients received BCPR and survived at the rate of $0.26,13$ additional patients would have survived to hospital discharge. The cost per year-of-life-saved by the EMS system with ETCPR would have been $\$ 5,233$ versus $\$ 8,886$ without ETCPR. The cost per additional year-of-lifesaved by ETCPR was $\$ 1580$.

Conclusion: This study found that ETCPR instruction by 9-1-1 dispatchers is a cost-effective addition to a two-tier, EMS system for the prehospital treatment of patients with ventricular fibrillation. 